\title{
Chemical Constituents of Artocarpus odoratissimus from Sarawak
}

\author{
Khong Heng Yen ${ }^{1 *}$, Nyotia Nyokat ${ }^{1}$, Clifford Junaidi Kutoi ${ }^{1}$, Ahmad Sazali Hamzah ${ }^{2}$, Isabel Fong Lim ${ }^{3}$ \\ ${ }^{1}$ School of Chemistry and Environmental Studies, Faculty of Applied Sciences, Universiti Teknologi MARA, 94300 Kota Samarahan, Sarawak, Malaysia. \\ ${ }^{2}$ Institute of Science, Universiti Teknologi MARA (UiTM), 40450 Shah Alam, Selangor, Malaysia. \\ ${ }^{3}$ Department of Paraclinical Sciences, Faculty of Medicine and Health Sciences, Universiti Malaysia Sarawak, Malaysia.
}

\section{ARTICLE INFO \\ Article history: \\ Received on: 03/08/2016 \\ Accepted on: 12/02/2017 \\ Available online: $30 / 08 / 2017$}

\section{Key words:}

Moraceae, Artocarpus odoratissimus, pinocembrin, pinostrobin, $\alpha$-amyrin acetate, $\beta$-amyrin acetate.

\begin{abstract}
Previous studies showed that Artocarpus species are rich in phenolic compounds, including flavonoids, stilbenoids and arylbenzofurons. Furthermore, compounds from Artocarpus species exhibited diverse biological activities including antibacterial, antitubercular, antiviral, antifungal, antiplatelet, antiarthritic, tyrosinase inhibitory and cytotoxicity. However, there is no phytochemical investigation on the Artocarpus odoratissimus from Sarawak. Thus, it would be interesting to develop a chemical profile of such Sarawakian species. Various chromatographic methods, such as liquid vacuum chromatography, radial chromatography and column chromatograpy were employed to isolate the chemical constituents from the different parts, namely root, bark and leaves of Artocarpus odoratissimus. The isolation and purification using different solvents system ratio of hexane and ethyl acetate (10:0, 8:2, 6:4, 4:6, 2:8, 0:10) and ethyl acetate and methanol (8:2) have led to two flavonoids; pinocembrin (1) and pinostrobin (2), and six triterpenoids; $\alpha$-amyrin acetate (3), $\beta$-amyrin acetate (4), traxateryl acetate (5), hexyl dodecanoate (6), $\beta$-sitosterol (7) and stigmasterol (8). The structures (1) - (8) were elucidated using various spectroscopic analysis, which included the Mass Spectroscopy (MS), Infrared (IR) spectroscopy, Ultra-violet (UV) spectroscopy and Nuclear Magnetic Resonance (NMR) spectroscopy, as well as by comparison with the reported data. Although the occurrence of compounds $\mathbf{1}$ and $\mathbf{2}$ were widely distributed in many of the plant species, but it was uncommon to the Artocarpus species which could be of chemotaxonomic significance to the genus Artocarpus.
\end{abstract}

\section{INTRODUCTION}

The genus Artocarpus belonging to the family of Moraceae consists of about 55 species. It is widely distributed throughout subtropical and tropical regions of the World from Indian subcontinent south of the Himalayas, Sri Lanka, Burma, Thailand, Indo-China, Southern China, Taiwan and Malay Peninsula (Kochummen, 2000). Artocarpus odoratissimus originated in Borneo. It is cultivated in the Philippines and is also found in the wild extensively in Brunei Darussalam, Sabah and Sarawak (Subhadrabandhu, 2001). Some Artocarpus species are used as traditional medicines. For example A. odoratissimus (terap) isused by local communities in Sarawak as an antidote

\footnotetext{
* Corresponding Author

Heng Yen Khong, School of Chemistry and Environmental Studies, Faculty of Applied Sciences, Universiti Teknologi MARA (UiTM), 94300 Kota Samarahan, Sarawak, Malaysia.

E-mail: khonghy@sarawak.uitm.edu.my
}

against centipede and scorpion stings by applying the ash from the leaves on the wounds, while for treatment of scabies, the ash is added with a little amount of coconut oil (Chai, 2006). The leaves can also be used to heal ulcers and burn. Besides, a decoction of the root is consumed for diarrhoea. Compounds from Artocarpus species exhibited diverse biological activities including antibacterial, antitubercular, antiviral, antifungal, antiplatelet, antiarthritic, tyrosinase inhibitory and cytotoxicity (Jagtap and Bapat, 2010). Previous studies showed that Artocarpus species are rich in phenolic compounds, including flavonoids, stilbenoids and arylbenzofurons. Ee et al. (2010) reported that the isolation of a new prenylated pyranoflavone derivatives, artosimmin and traxateryl acetate. Artosimmin exhibited significant cytotoxicity against cancer cell lines (HL-60 and MCF-7) and showed antioxidant properties. However, no phytochemical investigation or structural characterization on the Artocarpus odoratissimus from Sarawak has been reported to date, therefore it would be interesting to develop a chemical profile of such Sarawakian species. 


\section{MATERIAL AND METHODS}

\section{General Experimental Procedure}

Melting points were determined on Stuart model SMP10 melting points apparatus. Infrared spectra were recorded on Perkin Elmer FTIR Frontier model spectrophotometer; using potassium bromide (KBr) disc. GC-MS chromatograms were recorded using a Perkin Elmer Clarus 680 spectrometer.

The ${ }^{1} \mathrm{H}$ NMR spectra were recorded using Bruker 400 spectrometers which run at approximately $400 \mathrm{MHz}$ while ${ }^{13} \mathrm{C}$ NMR were measured using the same instrument and condition which run at approximately $100 \mathrm{MHz}$. Chemical shifts are given in $\delta(\mathrm{ppm})$ values relative to that of the solvents used. The column chromatography was performed using silica gel Merck Kieselgel 60 Art. No. 1.09385 .1000 of particle size $0.040-0.063 \mathrm{~mm} \mathrm{(230-}$ 400 mesh), silica gel $60 \mathrm{GF}_{254}$ (MERCK 1.007730) and silica gel 60 PF254 (MERCK 1.007749) were used for liquid vacuum and radial chromatography respectively.

\section{Plant Materials}

The leaves, bark and root of A. odoratissimus were collected from Kampung Mambong and Bau, Sarawak. Voucher specimen of the plant is kept in the School of Chemistry and Environmental Studies, Faculty of Applied Sciences at the University Teknologi MARA (UiTM) Sarawak, Samarahan Campus 2, Sarawak, Malaysia.

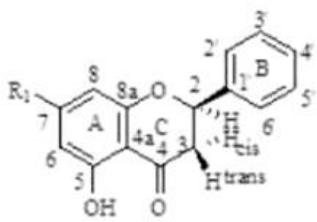

R1

$1 \mathrm{OH}$

$2 \mathrm{CH}_{3}$
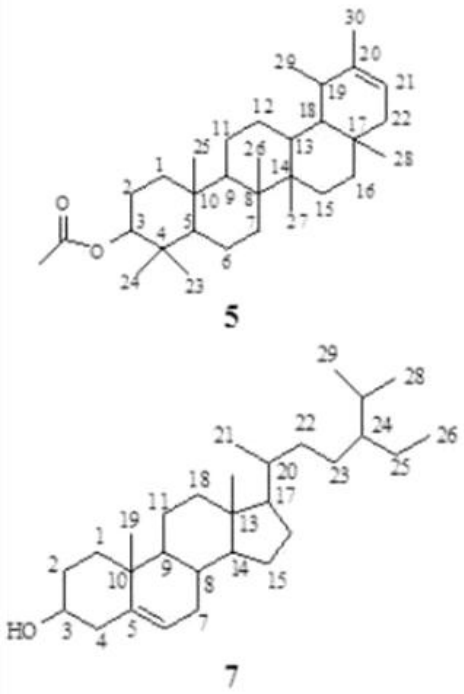

\section{Extraction and Isolation}

The air-dried leaves $(500.00 \mathrm{~g})$, stem bark (1097.06 g) and root (722.14 g) of A. odoratissimus were extracted by a cold extraction method for 24 hours and repeated thrice using n-hexane, ethyl acetate and methanol as solvents. The removal of solvents under reduced pressure gave different crude extracts, such as the nhexane, ethyl acetate and methanol crude extracts, respectively. The crude extracts were fractionated using silica gel vacuum liquid chromatography (VLC) with a stepwise gradient polarity solvents system such as hexane, hexane-ethyl acetate, ethyl acetatemethanol and methanol, followed by column chromatography to give major fractions. Fractions with the same $R_{f}$ values were combined and rechromatographed in appropriate solvent systems until pure isolates were obtained. The isolation and purification of crude ethyl acetate root extract using different solvents system ratio of hexane and ethyl acetate (10:0, 8:2, 6:4, 4:6, 2:8,0:10) and ethyl acetate and methanol (8:2) have led to two flavonoids; had afforded pinocembrin (1), pinostrobin (2) $\alpha$-amyrin acetate (3) and $\beta$-amyrin acetate (4) while the crude hexane bark extract had afforded traxateryl acetate (5) and hexyl dodecanoate (6). In addition, the leaves methanol extract had yielded $\beta$-sitosterol (7) and stigmasterol (8) (Figure 1)
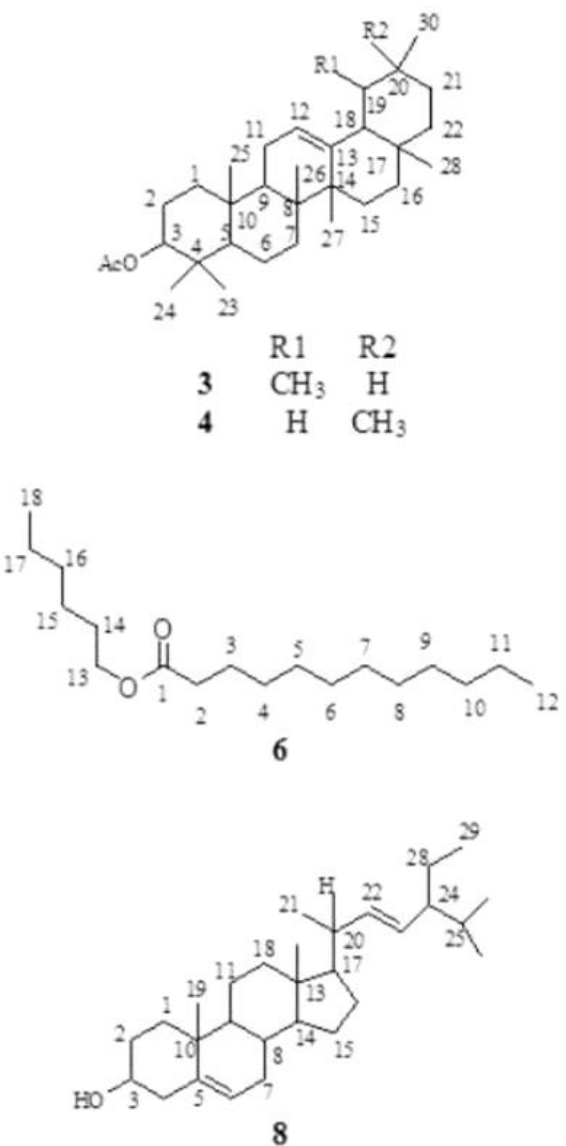

Fig. 1: structure of compounds (1-8) isolated from A. odoratissimus. 
int.); 256 (100) $\left[\mathrm{M}^{+} \mathrm{C}_{15} \mathrm{H}_{12} \mathrm{O}_{4}\right], 238$ (14), 179 (70), 152 (80), 124 (45), 104 (18), 77 (20), 69 (20) and 51(9); ${ }^{1} \mathrm{H}$ NMR (400 MHz; $\left.\mathrm{CDCl}_{3}\right): \delta 2.86$ (3-cis), 3.10 (3-trans), $5.43(2 \mathrm{H}, \mathrm{t}, \mathrm{J}=6.7), 6.03(\mathrm{H}$ 6), 6.03 (H-8), 7.41-7.49 (H-2'-H6'), 12.07 (C-5-OH). ${ }^{13} \mathrm{C}$ NMR (100 MHz; $\left.\mathrm{CDCl}_{3}\right): \delta 43.37$ (C-3), 79.18 (C-2), 95.56 (C-8), 96.81 (C-6), 102.97 (C-4a), 126.16 (C-2', C-6'), 128.87 (C-3', C4', C-5'), 138.42 (C-1'), 163.11 (C-8a), 164.34 (C-5), 195.65 (C4).

\section{Pinostrobin (2)}

Colorless crystal; mp $100-102^{0} \mathrm{C}$; IR $(\mathrm{KBr}) v_{\max } \mathrm{cm}^{-1}$ : 3467 (Ar-H stretching), 1741 ( $\mathrm{C}=\mathrm{O}$ ester), 1497, 1578 ( $\mathrm{C}=\mathrm{C}$ Aromatic), 1538, $1360(\mathrm{C}-\mathrm{O}), 968(=\mathrm{C}-\mathrm{H}) \mathrm{cm}^{-1}$; EI-MS m/z (\% rel. int.); 270 (90) $\left[\mathrm{M}^{+} \mathrm{C}_{16} \mathrm{H}_{14} \mathrm{O}_{4}\right], 269$ (50), 252 (8), 193 (100), 166 (80), 138 (50), 110 (18), 95 (35), 78 (15) and 51 (20);. ${ }^{1} \mathrm{H}$ NMR (400 MHz; $\mathrm{CDCl}_{3}$ ): $\delta 2.81$ (3-cis), 3.86 (C-7-OMe), 3.10 (3trans), $5.43(2 \mathrm{H}, \mathrm{t}, \mathrm{J}=6.7), 6.03(\mathrm{H}-8), 6.04(\mathrm{H}-6), 7.28-7.49(\mathrm{H}-2$ '$\mathrm{H} 6$ '), 12.07 (C-5-OH). ${ }^{13} \mathrm{C}$ NMR (100 MHz; $\mathrm{CDCl}_{3}$ ): $\delta 43.36$ (C3), 55.60 (C-7-OMe), 79.25 (C-2), 95.49 (C-6, C-8), 103.20 (C4a), 126.17 (C-2', C-6'), 128.92 (C-3', C-4', C-5'), 138.30 (C-1'), 163.16 (C-5), 164.36 (C-7), 164.63 (C-8a), 195.79 (C-4).

\section{a-Amyrin Acetate (3)}

White needles; $\mathrm{mp} 210-215^{\circ} \mathrm{C}$; IR (KBr) $v_{\max } \mathrm{cm}^{-1}: 2924$, 2853 (C-H stretching), 1734 (C=O ester), 1449, $1378 \quad\left(\mathrm{CH}_{3}\right.$ bending), and (C-O) $1243 \mathrm{~cm}^{-1}$; EI-MS m/z (\% rel. int.); 468 (1) $\left[\mathrm{M}^{+}, \mathrm{C}_{32} \mathrm{H}_{52} \mathrm{O}_{2}\right], 408$ (10), 365 (15), 219 (18), 218 (100), 203 (75), 189 (38), 175 (28); ${ }^{1} \mathrm{H}$ NMR (400 MHz; $\left.\mathrm{CDCl}_{3}\right): \delta 0.80(3 \mathrm{H}, \mathrm{s}, \mathrm{H}-$ 28), 0.87 (12H, s, H-23, H-24, H-25, H-26), 0.98 (3H, s, H-30), $1.01(3 \mathrm{H}, \mathrm{s}, \mathrm{H}-29), 1.07$ (3H, s, H-27), $2.05\left(3 \mathrm{H}, \mathrm{s}, \mathrm{CH}_{3}-\mathrm{CO}\right), 4.50$ $(1 \mathrm{H}, \mathrm{dd}, J=6,10, \mathrm{H}-3), 5.13(1 \mathrm{H}, \mathrm{t}, J=3.6, \mathrm{H}-12) ;{ }^{13} \mathrm{C}$ NMR $(100$ $\left.\mathrm{MHz} ; \mathrm{CDCl}_{3}\right): \delta 15.7(\mathrm{C}-25), 16.7(\mathrm{C}-26), 16.9(\mathrm{C}-24), 17.5(\mathrm{C}-$ 29), 18.3 (C-6), $21.3\left(\mathrm{CH}_{3}-\mathrm{CO}\right), 21.4$ (C-30), 23.2 (C-27), 23.4 (C11), 23.7 (C-2), 26.6 (C-16), 28.0 (C-23), 28.0 (C-28), 28.8(C-15), 31.3 (C-21), 32.9 (C-7), 33.8 (C-17), 36.8 (C-10), 37.7 (C-4), 38.5 (C-1), 39.6 (C-19), 39.7 (C-20), 40.0 (C-8), 41.5 (C-22), 42.1 (C14), 47.6 (C-9), 55.3 (C-5), 59.1 (C-18), 80.9 (C-3), 124.3 (C-12), 139.6 (C-13), $171.0\left(\mathrm{CH}_{3}-\mathrm{CO}\right)$.

\section{及-Amyrin Acetate (4)}

Colorless solid; mp 238-241 ${ }^{\circ} \mathrm{C}$; IR $(\mathrm{KBr}) v_{\max } \mathrm{cm}^{-1}$ : 2918, 2849 (C-H stretching), 1735 (C=O ester), 1452, $1377\left(\mathrm{CH}_{3}\right.$ bending), 1245 (C-O) $\mathrm{cm}^{-1}$; EI-MS m/z (\% rel. int.); 468 (10) [ $\mathrm{M}^{+}$, $\mathrm{C}_{32} \mathrm{H}_{52} \mathrm{O}_{2}$ ], 453 (2), 408 (1), 219 (18), 218 (100), 203 (25), 189 (11) and $175(5) ;{ }^{1} \mathrm{H}$ NMR (400 MHz; $\left.\mathrm{CDCl}_{3}\right): \delta 0.83(3 \mathrm{H}, \mathrm{s}, \mathrm{H}-$ 28), 0.87 (12H, s, H-23, H-24, H-29, H-30), 0.97 (6H, s, H-25, H26), 1.13 (3H, s, H-27), 2.05 (3H, s, $\left.\mathrm{CH}_{3}-\mathrm{CO}\right), 4.50(1 \mathrm{H}, \mathrm{m}, \mathrm{H}-3)$, $5.18(1 \mathrm{H}, \mathrm{t}, J=3.68, \mathrm{H}-12) ;{ }^{13} \mathrm{C} \mathrm{NMR}\left(100 \mathrm{MHz} ; \mathrm{CDCl}_{3}\right): \delta 15.6$ (C-25), 16.7 (C-26), 16.8 (C-24), $18.3(\mathrm{C}-6), 21.3\left(\mathrm{CH}_{3}-\mathrm{CO}\right), 23.2$ (C-11), 23.6 (C-2), 23.7 (C-30), 26.0 (C-27), 26.1 (C-15), 26.9 (C16), 28.0 (C-23), 28.4 (C-28), 31.1 (C-20), 32.5 (C-17), 32.6 (C7), 33.3 (C-29), 34.7 (C-21), 36.8 (C-10), 37.1 (C-22), 37.7 (C-4), 38.2 (C-1), 39.8 (C-8), 41.7 (C-14), 46.8 (C-19), 47.2 (C-18), 47.6
(C-9), 55.2 (C-5), 80.9 (C-3), 121.6 (C-12), 145.2 (C-13), 171.0 $\left(\mathrm{CH}_{3}-\underline{\mathrm{CO}}\right)$.

\section{Traxateryl Acetate (5)}

White needles; mp $239{ }^{\circ} \mathrm{C}$; IR (KBr) $v_{\max } \mathrm{cm}^{-1}: 2924$, 2852 (C-H stretching), 1733 (C=O ester), 1449, $1366\left(\mathrm{CH}_{3}\right.$ bending), and (C-O) $1243 \mathrm{~cm}^{-1}$; EI-MS m/z (\% rel. int.); 468 (1) $\left[\mathrm{M}^{+}, \mathrm{C}_{32} \mathrm{H}_{52} \mathrm{O}_{2}\right], 408$ (10), 365 (15), 219 (18), 203 (75), 189 (38) and $175(28) ;{ }^{1} \mathrm{H}$ NMR $\left(400 \mathrm{MHz} ; \mathrm{CDCl}_{3}\right): \delta 0.81(6 \mathrm{H}, \mathrm{s}, \mathrm{H}-25 \&$ 28), 0.88 (3H, s, H-24), 0.89 (3H, s, H-30), 0.93 (3H, s, H-29), 0.99 (3H, s, H-27), 1.02 (3H, s, H-23), 1.08 (3H, s, H-26), 4.52 $(1 \mathrm{H}, \mathrm{m}, \mathrm{H}-3), 5.13(1 \mathrm{H}, \mathrm{t}, \mathrm{J}=3.68 \mathrm{~Hz}) ;{ }^{13} \mathrm{C} \mathrm{NMR}(100 \mathrm{MHz}$; $\mathrm{CDCl}_{3}$ ): $\delta 15.8$ (C-27), 16.8 (C-24), 16.9 (C-25), 17.5 (C-28), 18.2 (C-6), $21.3\left(\mathrm{CH}_{3}-\mathrm{CO}\right), 21.4$ (C-29), 23.2 (C-26), 23.4 (C-12), 23.6 (C-2), 26.6 (C-15), 28.1 (C-11), 28.1 (C-30), 28.7 (C-23), 31.2 (C7), 32.8 (C-16), 33.7 (C-17), 36.8 (C-10), 37.7 (C-4), 38.4 (C-1), 39.6 (C-13), 39.6 (C-19), 40.0 (C-14), 41.5 (C-22), 42.0 (C-8), 47.6 (C-9), 55.2 (C-5), 59.0 (C-18), 80.9 (C-3), 124.3 (C-21), 139.6 (C-20), 170.9 (CH3-CO).

\section{Hexyl Dodecanoate (6)}

White solid; mp 61-62 ${ }^{\circ} \mathrm{C}$, IR (KBr) $v_{\max } \mathrm{cm}^{-1}: 2924$ (C$\mathrm{H}$ stretching), 1735 (C=O ester), 1456, $1369\left(\mathrm{CH}_{3}\right.$ bending), and (C-O) $1242 \mathrm{~cm}^{-1}$; EI-MS m/z (\% rel. int.); 284 (15) $\left[\mathrm{M}^{+}\right.$ $\mathrm{C}_{18} \mathrm{H}_{36} \mathrm{O}_{2}$.], 241 (10), 157 (15), 101 (35), 88 (100), 73 (18) and 55 (28); ${ }^{1} \mathrm{H}$ NMR (400 MHz; $\left.\mathrm{CDCl}_{3}\right): \delta 0.88(3 \mathrm{H}, \mathrm{t}, J=6.6 \mathrm{~Hz}), 0.91$ $(3 \mathrm{H}, \mathrm{t}, J=6.8), 1.27-1.45(22 \mathrm{H}, \mathrm{m}), 1.57-1.65(4 \mathrm{H}, \mathrm{m}), 2.31(2 \mathrm{H}$, $\mathrm{t}, J=7.4), 4.06(2 \mathrm{H}, \mathrm{t}, \mathrm{J}=6.7) ;{ }^{13} \mathrm{C} \mathrm{NMR}\left(100 \mathrm{MHz} ; \mathrm{CDCl}_{3}\right): \delta 14.1$ (C-12), 14.1 (C-18), 22.7 (C-11), 22.7 (C-17), 25.0 (C-3), 25.6 (C15), 28.6 (C-14), 29.2 (C-4), 29.3 (C-5), 29.3 (C-9), 29.5 (C-7), 29.6 (C-6), 29.6 (C-8), 31.9 (C-10), 31.9 (C-16), 34.4(C-2), 64.4 (C-13), 174.1 (C-1).

\section{$\beta$-Sitosterol (7)}

White needles; $\mathrm{mp} 145-147{ }^{\circ} \mathrm{C}$; IR $(\mathrm{KBr}) v_{\max } \quad \mathrm{cm}^{-1}: 3436$ $(\mathrm{OH}), 2937(\mathrm{C}-\mathrm{H}), 2867,1063(\mathrm{C}=\mathrm{C})$, and $\left(-\mathrm{CH}_{3}\right) 1454,1382, \mathrm{~cm}$ ${ }^{1}$; EI-MS m/z (\% rel. int.); 414 (55) [ $\left.\mathrm{M}^{+}, \mathrm{C}_{29} \mathrm{H}_{50} \mathrm{O}\right], 394$ (24), 381 (17), 354 (5), 329 (34), 303 (23), 273 (10), 255 (23), 231 (15), 213 (27), 199 (12), 187 (10), 173 (15), 159 (28), 145 (34), 133 (29), 119 (29), 107 (48), 95 (48), 81 (56), 57 (59), 43 (100), 41 (56); ${ }^{1} \mathrm{H}$ NMR (400 MHz; $\left.\mathrm{CDCl}_{3}\right): \delta$ 0.68-1.05 (18H, s, $\left.6 \mathrm{X} \mathrm{CH}_{3}\right), 1.05-$ $2.36\left(22 \mathrm{H}, \mathrm{m}, 11 \times \mathrm{CH}_{2}\right), 3.53(1 \mathrm{H}, \mathrm{m}, \mathrm{H}-3), 5.35(1 \mathrm{H}, \mathrm{d}, \mathrm{J}=$ 5.1Hz, H-6). ${ }^{13} \mathrm{C}$ NMR $\left(100 \mathrm{MHz} ; \mathrm{CDCl}_{3}\right): \delta 11.8(\mathrm{C}-18), 12.0(\mathrm{C}-$ 29), 18.8 (C-21), 19.0 (C-27), 19.4 (C-19),19.8 (C-26), 21.1 (C11), 23.0 (C-28), 24.3 (C-15), 26.0 (C-23), 28.2 (C-16), 29.1 (C25), 31.6 (C-2), 31.9 (C-7), 31.9 (C-8), 33.9 (C-22), 36.1 (C-20), 36.5 (C-10), 37.2 (C-1), 39.7 (C-12), 42.3 (C-4), 42.3 (C-13), 45.8 (C-24), 50.1 (C-9), 56.0 (C-17), 56.7 (C-14), 71.8 (C-3), 121.7 (C6) and $140.7(\mathrm{C}-5)$.

\section{Stigmasterol (8)}

White needles;mp145-147 ${ }^{\circ} \mathrm{C}$; IR (KBr) $v_{\max } \mathrm{cm}^{-1}: 3436$ (OH), $2937(\mathrm{C}-\mathrm{H}), 1708(\mathrm{C}=\mathrm{C})$, and $\left(-\mathrm{CH}_{3}\right)$ 1454, 1382, $\mathrm{cm}^{-1}$; EIMS m/z (\% rel. int.);412 [ $\left.\mathrm{M}^{+}, \mathrm{C}_{29} \mathrm{H}_{48} \mathrm{O}\right], 271,255,159,133,81$ 
and 55; ${ }^{1} \mathrm{H}$ NMR (400 MHz; $\left.\mathrm{CDCl}_{3}\right): \delta 0.72(3 \mathrm{H}, \mathrm{s}, \mathrm{Me}-18), 0.83$ $(3 \mathrm{H}, t, J=7.2 \mathrm{~Hz}, \mathrm{Me}-29), 0.85(3 \mathrm{H}, d, J=6.9 \mathrm{~Hz}, \mathrm{Me}-27), 0.88$ $(3 \mathrm{H}, d, J=6.9 \mathrm{~Hz}, \mathrm{Me}-26), 1.03$ (3H, $s, \mathrm{Me}-19), 1.03(3 \mathrm{H}, d, J=6.9$ $\mathrm{Hz}, \mathrm{Me}-21), 3.54(1 \mathrm{H}, m, \mathrm{H}-3), 5.03(1 \mathrm{H}, d d, J=8.4,15.0 \mathrm{~Hz}, \mathrm{H}-$ 21), $5.17(1 \mathrm{H}, d d, J=8.4,15.0 \mathrm{~Hz}, \mathrm{H}-21), 5.46(1 \mathrm{H}, b r d, \mathrm{H}-6) ;{ }^{13} \mathrm{C}$ NMR (100 MHz; $\mathrm{CDCl}_{3}$ ): $\delta 12.0$ (C-18), 12.2 (C-29), $19.0(\mathrm{C}-$ 27), 19.4 (C-26), 21.0 (C-19), 21.1 (C-11), 21.2 (C-21), 24.4 (C15), 25.4 (C-28), 28.9 (C-16), 31.7 (C-2), 31.7 (C-7), 31.9 (C-8), 31.9 (C-25), 36.5 (C-10), 37.3 (C-1), 39.7 (C-12), 40.5 (C-20), 42.2 (C-13), 42.3 (C-4), 50.2 (C-9), 51.3 (C-24), 56.0 (C-17), 56.9 (C-14), 71.8 (C-3), 121.7 (C-6), 129.3 (C-23), 138.3 (C-22) and $140.2(\mathrm{C}-5)$.

\section{RESULTS AND DISCUSSION}

The extraction and isolation from different parts of Artocarpus odoratissimus have led to eight compounds (Figure 1). The identification of pure compounds was carried out using spectroscopic methods: Infrared (IR) spectroscopy, Nuclear Magnetic Resonance (NMR) spectroscopy and Gas Chromatography Mass Spectroscopy (GC-MS) and a comparison with the reported data. From the root extract, afforded two flavonoids, which were identified and characterized as pinocembrin (1) and pinostrobin (2) based on data comparison literature. As for the two triterpenoids isolated, identified as $\alpha$-amyrin acetate (3) and $\beta$-amyrin acetate (4). Compound (3) and (4) were deduced based on the spectral evidence and by comparison of spectral data with literature values (Ogihara et al., 2000). Two compounds isolated and elucidated from the bark are traxateryl acetate (5) and hexyl dodecanoate (6). Compound (5) have been found and was report before by Ee et al., (2010). The structural elucidation of (6) was based on the spectroscopic data and by comparison of the data with the literature (Shimizu et al., 2012). A chloroform-soluble fraction from a methanol extract of leaves was subjected to column chromatography on silica gel to give $\beta$-Sitosterol (7) and stigmasterol (8). The structural elucidations of these compounds were based on their spectroscopic data and by comparison of these data with the literature (Ragasa $e t$ al., 2014). Based on the literature, these compounds have revealed diverse bioactivities; Pinocembrin (1) is one of the primary flavonoids isolated from the variety of plants, mainly from Pinus heartwood, Eucalyptus, Populus, Euphorbia, and Sparattosperma leucanthum, in the diverse flora and purified by various chromatographic techniques.

Pinocembrin is a major flavonoid molecule incorporated as multifunctional in the pharmaceutical industry. Its vast range of pharmacological activities has been well researched including antimicrobial, anti-inflammatory, antioxidant, and anticancer activities. In addition, pinocembrin can be used as neuroprotective against cerebral ischemic injury with a wide therapeutic time window, which may be attributed to its antiexcitotoxic effects (Rasul et al., 2013). Pinostrobin (2) was isolated from Polygonum lapathifolium nodosum quickly penetrates through cytoplasm to the cellular nucleus of the cultured cells, and gives intensive apoptotic response in stimulating leukemic cells in vitro (Smolarz et al., 2006). With increasing concentration, pinostrobin caused a gradual leakage, also contributing to breakage of the envelope and virus inactivation. Treatment effect of oral pinostrobin in vivo showed that pinostrobin possesses definite therapeutic effect in the development of lesion score (Wu et al., 2011).

Previous studies indicated that $\alpha$-amyrin acetate (3) not only improves glucose tolerance in normal rats significantly, but also lowers the blood glucose profile in STZ-induced diabetic rats and $\mathrm{db} / \mathrm{db}$ mice. It also improves atherogenic lipid profiles and increases HDL-C levels significantly. These results suggest that compound (3) can be used as an effective antidiabetic cum lipid lowering agent for type 2 diabetes mellitus patients (Singh et al., 2009). $\beta$-amyrin acetate (4) isolated from the Alstonia boonei stem bark exhibited profound anti-inflammatory activity (Okoye $e t$ al., 2014).

Triterpenes (3) and (4) were also reported to exhibit sedative, anxiolytic and anticonvulsant properties (Aragao et al., 2009). $\beta$-Sitosterol (7) was observed to have growth inhibitory effects on human breast MCF-7 and MDA-MB-231 adenocarcinoma cells (Awad et al., 2007). Stigmasterol (8) isolated from $B$. monosperma could reduce the levels of serum triiodothyronine $\left(\mathrm{T}_{3}\right)$ and/or thyroxin $\left(\mathrm{T}_{4}\right)$ in mice. Its administration at $2.6 \mathrm{mg} / \mathrm{kg} / \mathrm{d}$ for 20 days reduced serum triiodothyronine $\left(\mathrm{T}_{3}\right)$, thyroxin $\left(\mathrm{T}_{4}\right)$ and glucose concentrations as well as the activity of hepatic glucose-6-phophatase (G-6-Pase) with a concomitant increase in insulin indicating its thyroid inhibiting and hypoglycemic properties. A decrease in the hepatic lipid peroxidation (LPO) and an increase in the activities of catalase (CAT), superoxide dismutase (SOD) and glutathione (GSH) suggested its antioxidative potential. The highest concentration tested $(5.2 \mathrm{mg} / \mathrm{kg})$ evoked pro-oxidative activity (Panda et al., 2009).

\section{CONCLUSIONS}

Phytochemical study on A. odoratissimus had successfully isolated eight pure compounds; pinocembrin (1), pinostrobin (2), $\alpha$-amyrin acetate (3) and $\beta$-amyrin acetate (4) were isolated from the root of A. odoratissimus, traxateryl acetate (5) and hexyl dodecanoate (6) from the bark of A. odoratissimus whereas $\beta$-sitosterol (7) and stigmasterol (8) from the leaves. In addition, compounds 1- 4 and 6 from A. odoratissimus were reported for the first time.

Although the occurrence of compounds $\mathbf{1}$ and $\mathbf{2}$ were widely distributed in many of the plant species, but it was uncommon to the Artocarpus species which could be of chemotaxonomic significance to the genus Artocarpus in particular, and also to the family Moraceae.

\section{ACKNOWLEDGEMENTS}

The authors would like to acknowledge the financial assistance provided by the Ministry of Higher Education, under Research Acculturation Grant Scheme entitled: Antiinflammatory, Antioxidant And Antimicrobial Activities of 
Artocarpus odoratissimus (600-RMI/RAGS 5/3 (95/2013). The authors also extended their thanks to Research Management Institute, Universiti Teknologi MARA (UiTM), Malaysia for their excellent support and assistance.

Conflict of Interests: There are no conflicts of interest.

\section{REFERENCES}

Aragãoa GF, Carneiroa LM, Juniora AP, Bandeirab PN, Lemosb TL, Viana GS. Evidence for excitatory and inhibitory amino acids participation in the neuropharmacological activity of alpha-and betaamyrin acetate. Open Pharmacol J, 2009;3:9-16.

Awad AB, Chinnam M, Fink CS, Bradford PG. $\beta$-Sitosterol activates Fas signaling in human breast cancer cells. Phytomedicine, 2007;14(11):747-54.

Chai, PK. Medicinal plants of Sarawak. Lee Ming Press, Kuching, 2006; 212pp.

Ee GCL, Teo SH, Rahmani M, Lim CK, Lim YM, Bong FJC. Artosimmin-A Potential Anti-Cancer Lead Compound from Artocarpus odoratissimus. Lett Org Chem, 2010;7(3), 240-244.

Jagtap UB, Bapat VA.Artocarpus: A review of its traditional uses, phytochemistry and pharmacology. J Ethnopharmacol, 2010; 129: $142-166$.

Kochummen KM, Go R. Ficus L. Moraceae. Tree Flora of Sabah and Sarawak, 2000; 3:213-317.

Ogihara $\mathrm{K}$, Nishizato $\mathrm{S}$, Ishigaki $\mathrm{M}$, Higa $\mathrm{M}$, Yogi $\mathrm{S}$. Triterpenoids and Phytosteroids from the Leaves of Cynanchum liukiuense. Bull. Fac. Sci. Univ. Ryukyus, 2000; 83-92.

Okoye NN, Ajaghaku DL, Okeke HN, Ilodigwe EE, Nworu CS, Okoye FB. beta-Amyrin and alpha-amyrin acetate isolated from the stem bark of Alstonia boonei display profound anti-inflammatory activity. PharmBiol, 2014;52(11):1478-86.

Panda S, Jafri M, Kar A, Meheta BK. Thyroid inhibitory, antiperoxidative and hypoglycemic effects of stigmasterol isolated fromButeamonosperma. Fitoterapia, 2009; 80(2):123-6.
Pessini GL, Dias Filho BP, Nakamura C V, Cortez DAG. Antibacterial activity of extracts and neolignans from Piper regnellii (Miq.) C. DC. var. pallescens (C. DC.) Yunck. MemInst Oswaldo Cruz, 2003;98(8):1115-1120.

Ragasa CY, Caro JL, Shen CC.Triterpenes and Sterol from Artocarpus ovatus. J Appl Pharm Sci, 2014a;4(10):7-11.

Rasul A, Millimouno FM, Ali Eltayb W, Ali M, Li J, Li X. Pinocembrin: a novel natural compound with versatile pharmacological and biological activities. Biomed Res Int, 2013; 2013. http://dx.doi.org/10.1155/2013/379850.

Singh AB, Yadav DK, Maurya $R$, Srivastava AK. Antihyperglycaemic activity of $\alpha$-amyrin acetate in rats and $\mathrm{db} / \mathrm{db}$ mice. Natural product research, 2009; 23(9): 876-82.

Shimizu N, Kuwahara Y, Yakumaru R, Tanabe T. "n-Hexyl laurate and fourteen related fatty acid esters: new secretory compounds from the julid millipede, Anaulaciulus sp. J Chem Ecol, 2012;38(1): 2328 .

Smolarz HD, Mendyk E, Bogucka-Kocka A, Kockic J. Pinostrobin-an anti-leukemic flavonoid from Polygonum lapathifolium $L$. ssp. nodosum (Pers.) Dans. Z Naturforsch C, 2006;61(1-2):64-8.

Subhadrabandhu S. 2001. Under-utilized tropical fruits of Thailand (English): Thailand. Regional Office for Asia and the Pacific.

Wu N, Kong Y, Zu Y, Fu Y, Liu Z, Meng R, Liu X, Efferth T. Activity investigation of pinostrobin towards herpes simplex virus-1 as determined by atomic force microscopy. Phytomedicine, 2011;18(2):110-

\section{How to cite this article:}

Khong HY, Nyokat N, Kutoi CJ, Hamzah AS, Fong IL. Chemical Constituents of Artocarpus odoratissimus from Sarawak. J App Pharm Sci, 2017; 7 (08): 137-141. 